Document downloaded from:

http://hdl.handle.net/10251/103501

This paper must be cited as:

Martín Furones, AE.; Anquela Julián, AB.; Dimas-Pagés, A.; Cos-Gayón López, FJ. (2017). Computational time reduction for sequential batch solutions in GNSS precise point positioning technique. Computers \& Geosciences. 105:34-42.

doi:10.1016/j.cageo.2017.03.023

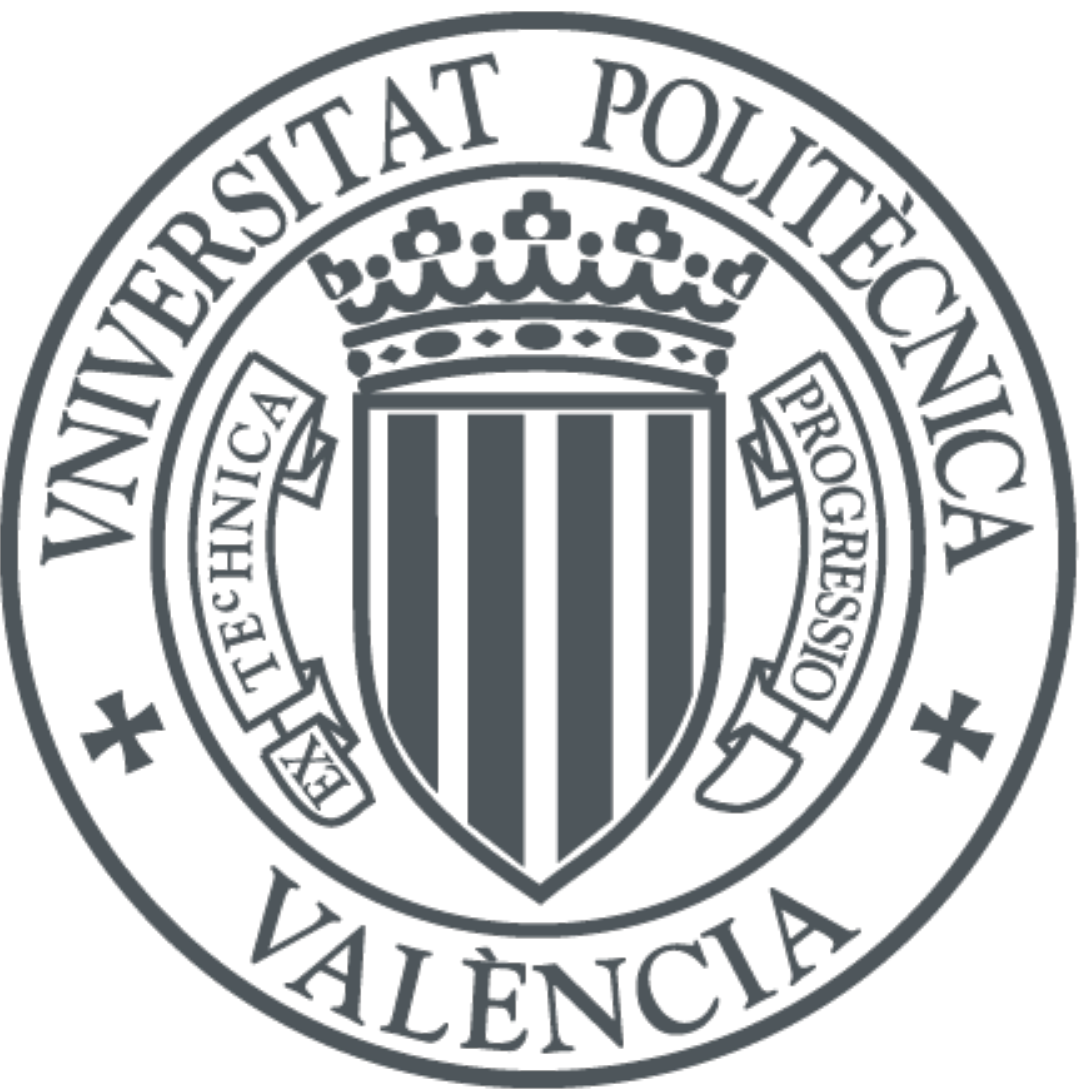

The final publication is available at

https://doi.org/10.1016/j.cageo.2017.03.023

Copyright Elsevier

Additional Information 


\title{
Computational time reduction for sequential batch solutions in GNSS precise point positioning technique
}

\author{
Angel Mart n ${ }^{\mathrm{a}}$, , Ana Bel n Anquela Juli n ${ }^{\mathrm{a}}$, Alejandro Dimas-Pages ${ }^{\mathrm{b}}$, Fernando Cos-Gay $\mathrm{n}^{\mathrm{c}}$ \\ ${ }^{a}$ Department of Cartographic Engineering, Geodesy and Photogrammetry, Universitat Polit cnica de Val ncia, C/Camino de Vera s/n, 46022 Valencia, Spain \\ b Institut Cartogr fic Valenci, Ciutat Administrativa 9 D' Octubre-Torre 1, Carrer de Castan Tobe as, 77, 46022 Valencia, Spain \\ ${ }^{c}$ Department of Architectural Constructions, Universitat Polit cnica de Val ncia, C/Camino de Vera s/n, Valencia 46022, Spain
}

\section{A R T I C L E I N F O}

\section{Keywords:}

Precise point positioning

Batch processing

Computational time reduction

\begin{abstract}
A B S T R A C T
Precise point positioning (PPP) is a well established Global Navigation Satellite System (GNSS) technique that only requires information from the receiver (or rover) to obtain high-precision position coordinates. This is a very interesting and promising technique because eliminates the need for a reference station near the rover receiver or a network of reference stations, thus reducing the cost of a GNSS survey.

From a computational perspective, there are two ways to solve the system of observation equations produced by static PPP either in a single step (so-called batch adjustment) or with a sequential adjustment/filter. The results of each should be the same if they are both well implemented. However, if a sequential solution (that is, not only the final coordinates, but also those observed in previous GNSS epochs), is needed, as for convergence studies, finding a batch solution becomes a very time consuming task owing to the need for matrix inversion that accumulates with each consecutive epoch. This is not a problem for the filter solution, which uses information computed in the previous epoch for the solution of the current epoch. Thus filter implementations need extra considerations of user dynamics and parameter state variations between observation epochs with appropriate stochastic update parameter variances from epoch to epoch. These filtering considerations are not needed in batch adjustment, which makes it attractive.

The main objective of this research is to significantly reduce the computation time required to obtain sequential results using batch adjustment. The new method we implemented in the adjustment process led to a mean reduction in computational time by $45 \%$.
\end{abstract}

\section{Introduction}

Since the pioneering work of Zumberge et al. (1997), precise point positioning (PPP) has been studied extensively (Kouba and $\mathrm{H}$ roux, 2001; Gao and Shen, 2001; Colombo et al., 2004; Bisnath et al., 2008; Chen et al., 2009; Geng et al., 2010) and applied to a wide variety of potential applications both in static and kinematic environments, including not just positioning and navigation, but also plate tectonics studies, resource management in remote areas, aerial photogrammetry and sea-level measurements (Chen, 2004; Heroux et al., 2004; Kouba, 2005; Zhang and Andersen, 2006; Bisnath and Gao, 2008).

PPP uses high-precision carrier phase and pseudorange observations of a single receiver in processing undifferenced Global Naviga- tion Satellite System (GNSS) algorithms, in which the most accurate satellite orbits and clock information as published by the International GNSS service (IGS), (Dow et al., 2009; Ray, 2010), are used.

The undifferenced GNSS observation equations for pseudorange and carrier phase measurements are based on the following simplified observation equations (Leick, 2004; Hofmann-Wellenhof et al., 2008):

$$
\begin{aligned}
& P\left(L_{i}\right)=\rho+c(d t-d T)+d_{\text {trop }}+d_{\frac{\text { Ion }}{L_{i}}}+\varepsilon \\
& \phi\left(L_{i}\right)=\rho+c(d t-d T)+d_{\text {trop }}-d_{\frac{\text { Ion }}{L_{i}}}+\lambda_{i} N_{i}+\varepsilon
\end{aligned}
$$

where $P\left(L_{i}\right){ }_{i}$ s the measured pseudorange on $L_{i}$ frequency (m); $\left(L_{i}\right)$ ${ }_{i} \mathrm{~S}$ the measured carrier phase on $L_{i}(\mathrm{~m}) ; \quad$ is the geometric range between the receiver and the satellite $(\mathrm{m})$; $c$ is the speed of light (m/

Corresponding author.

Email addresses: aemartin@upvnet.upv.es (A. Martín); anquela@cgf.upv.es (A.B. Anquela Julián); aldimpa@gmail.com (A. Dimas-Pages); fcosgay@csa.upv.es (F. Cos-Gayón) 
s); $d T$ is the satellite clock bias with respect to Global Positioning System (GPS) time (s); $d t$ is the receiver clock bias respect to the GPS time (s); $d_{\text {Trop }}$ is the tropospheric delay (m); $d_{\text {Ion/Li }}$ is the ionosphere delay on $L_{i}(\mathrm{~m}) ;{ }_{i i} \mathrm{~s}$ the carrier wavelength on $L_{i}(\mathrm{~m} /$ cycle $) ; N_{i}$ is the integer phase ambiguity on $L_{i}$ (cycles); and represents all remaining biases including the measurement noise and multipath effect in pseudorange or carrier phase (m).

Finally:

$\rho=\sqrt{\left(X_{S}-X\right)^{2}+\left(Y_{S}-Y\right)^{2}+\left(Z_{S}-Z\right)^{2}}$

where $\left(X_{S}, Y_{S}, Z_{S}\right)$ represents the satellite position and $(X, Y, Z)$ represents the receiver position, both in the Earth-fixed geocentric reference frame.

Linear combinations from the two frequencies can reduce or even eliminate some of the parameters presented in the previous equations. One of these is the ionosphere-free combination, which eliminates the first-order delay (more than the $99 \%$ of the total delay) of the ionosphere (Seepard and Bisnath, 2014). This combination is traditionally used as a functional model for PPP. IGS precise satellite clocks are estimated from the ionosphere-free combination of carrier phase and pseudorange observations using a world wide network solution (Datch el al. 2007), therefore, they contain the ionosphere-free combination of the satellite electronic biases, so no differential code bias (DCB) correction is needed for the ionosphere-free linear combination of P(L1), P(L2) data.

The ionosphere-free pseudorange $\left(P\left(L_{I F}\right)\right)$ and carrier phase $\left(\left(L_{I F}\right)\right)$ combinations can be formed as follows (Leick, 2004; Hofmann-Wellenhof et al., 2008):

$$
\begin{aligned}
& P\left(L_{I F}\right)=\frac{f_{1}^{2} P\left(L_{1}\right)-f_{2}^{2} P\left(L_{2}\right)}{f_{1}^{2}-f_{2}^{2}}=\rho+c(d t-d T)+d_{\text {trop }}+\varepsilon \\
& \phi\left(L_{I F}\right)=\frac{f_{1}^{2} \phi\left(L_{1}\right)-f_{2}^{2} \phi\left(L_{2}\right)}{f_{1}^{2}-f_{2}^{2}}=\rho+c(d t-d T)+d_{\text {trop }}+N_{I F}+\varepsilon
\end{aligned}
$$

where $f_{1}$ and $f_{2}$ are the GPS frequencies on $L_{1}$ and $L_{2}(\mathrm{~Hz})$, respectively, and $N_{I F}$ is the ionosphere-free ambiguity term, further described below:

$N_{I F}=\frac{c f_{1} N_{1}-c f_{2} N_{2}}{f_{1}^{2}-f_{2}^{2}}$

It should be noted that the ionosphere-free ambiguity term is no longer an integer number. Ambiguity resolution is an important aspect in PPP, a review of the principal methods can be found in Shi and Gao (2015); in the present research it is treated as a floating-point number.

To transform point positioning to PPP, high-accuracy satellite coordinates and clock bias are needed. Therefore, the best approach to solving these system equations, in a post-process stage, is to use the IGS final orbit and clock products (Dow et al., 2009), which are freely available for public use. These products are made available to the user with a lag time of 1218 days, (IGS 2013). In recent years, the accuracy of IGS orbit and clock products has improved drastically (Dow et al., 2009), the orbit accuracy of the final IGS products is better than $2.5 \mathrm{~cm}$, and the clock accuracies are approximately 75 ps.

The system of observation equations outlined above should be soved with a least-squares adjustment. This can be done in a single step (batch adjustment) or with a sequential adjustment/filter. The disadvantage of batch adjustment is that it may become too computationally time consuming even for powerful computers, especially for a large set of equations.

Additionally, extra computational time is required if a sequential solution (coordinates of the receiver at each epoch) are required in batch adjustment. This sequential solution is essential for studies of the convergence time required for PPP; thus, this research focuses on reducing the computation time required for a sequential PPP solution using batch adjustment to solve system equations.

As an example, a batch adjustment using $6 \mathrm{~h}$ of GNSS observation at 30-s intervals, 8 visible satellites per epoch, and 2 observation equations per epoch per satellite with no cycle slips will generate a final system with 11,520 observation equations and 3 station coordinate parameters (in the static case), 720 receiver clock parameters, 3 troposphere parameters (one every two hours) and the ambiguity terms for each observed satellite. If we consider only the ambiguity for the same 8 satellites for the entire observation period, this system of equations has a total of 732 parameters to solve; if a sequential solution is required, a different system equation need to be solved at each epoch, and so, in this example, 720 growing different system equations should be solved. Taking into account that the computing time for matrix inversion is increasing quadratically with the size of the matrix, a procedure to reduce the time in the matrix inversion is key for reducing the sequential processing time in batch adjustment solution.

This manuscript is written as follows: Section 2 explains the classical PPP software development, Section 3 introduces the new strategy to reduce the computational time in the sequential batch processing, Section 4 explains in detail how the design matrices should be formed in accordance with the new strategy, in Section 5 the experiments to check the proposed method are explained and, finally, a brief concluding section ends the paper.

\section{PPP software}

A PPP sequential batch approach was implemented using MATLAB software. To achieve the highest possible PPP positioning accuracy, phase wind-up, antenna phase offset and variation at the satellite and receiver, solid Earth tide, pole tide, relativistic corrections, and pseudorange- and carrier-phase biases are modelled in accordance with international standards (Kouba and H roux, 2001); if a cycle slip was detected, a new column for the satellite (a new ambiguity term) was introduced in the design matrix. Finally, the developed software (UPV software) can be considered to be the state-of-the-art in PPP. Fig. 1 shows the receiver coordinates differences between the BERNESE 5.2 software solution (Dach et al., 2015) and the final IGS weekly coordinates for JPLM permanent IGS station in static mode using the data from the first $6 \mathrm{~h}$ of October 1, 2013. Stacking equations into blocks of ten minutes are used for the coordinates comparisons; the same differences are plotted for the results of the developed software.

The core of the developed software is the establishment of the observation equations (the matrix definition $A$ ), because its solution is quite simple using MATLAB libraries and least-squares theory:

$A x=b+v$

where $A$ is the design matrix of coefficients; $x$ is the unknowns or parameter vector, $b$ is the vector of observations (observed minus computed terms) and $v$ is observation noise and uncertainty (the so-called residuals), which are assumed to be Gaussian normally distributed with zero mean.

The strategy for solving Eq. (3) is to minimize the sum of the squares of the residuals, obtaining the common notation:

$x=\left(A^{T} W A\right)^{-1}\left(A^{T} W b\right)$

where $W$ is introduced as the weight matrix, which is diagonal, considering uncorrelated observations. 


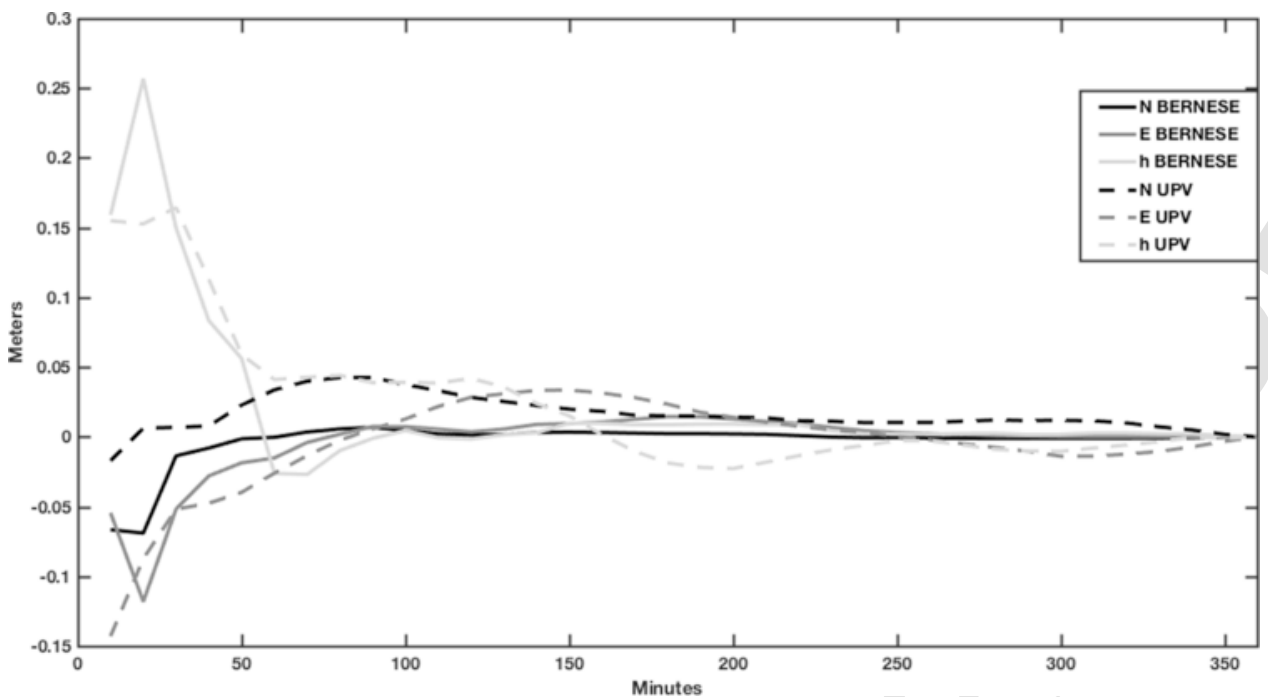

Fig. 1. Precise point positioning coordinate convergence (in comparison with the weekly IGS coordinates) of the JPLM IGS GNSS permanent station using BERNESE 5.2 and UPV software. North (N), East (E) and Up (h) components are considered.

This system can be simplified to:

$x=\left(A^{T} A\right)^{-1}\left(A^{T} b\right)$

if the design matrices are renamed using the notation: $A=\sqrt{W} A$ and $b=\sqrt{W} b$. In this case $W$ is a diagonal matrix, so the square root of the matrix is simply the square root of the elements of the diagonal.

The linearization of the observation equations is the basis for the construction of the design matrix (Hofmann-Wellenhof et al., 2008). An example of PPP design matrix from $t_{1}$ to $t_{n}$ epochs and observed satellites 1 to $S$, is:

\section{Coordinate columns}

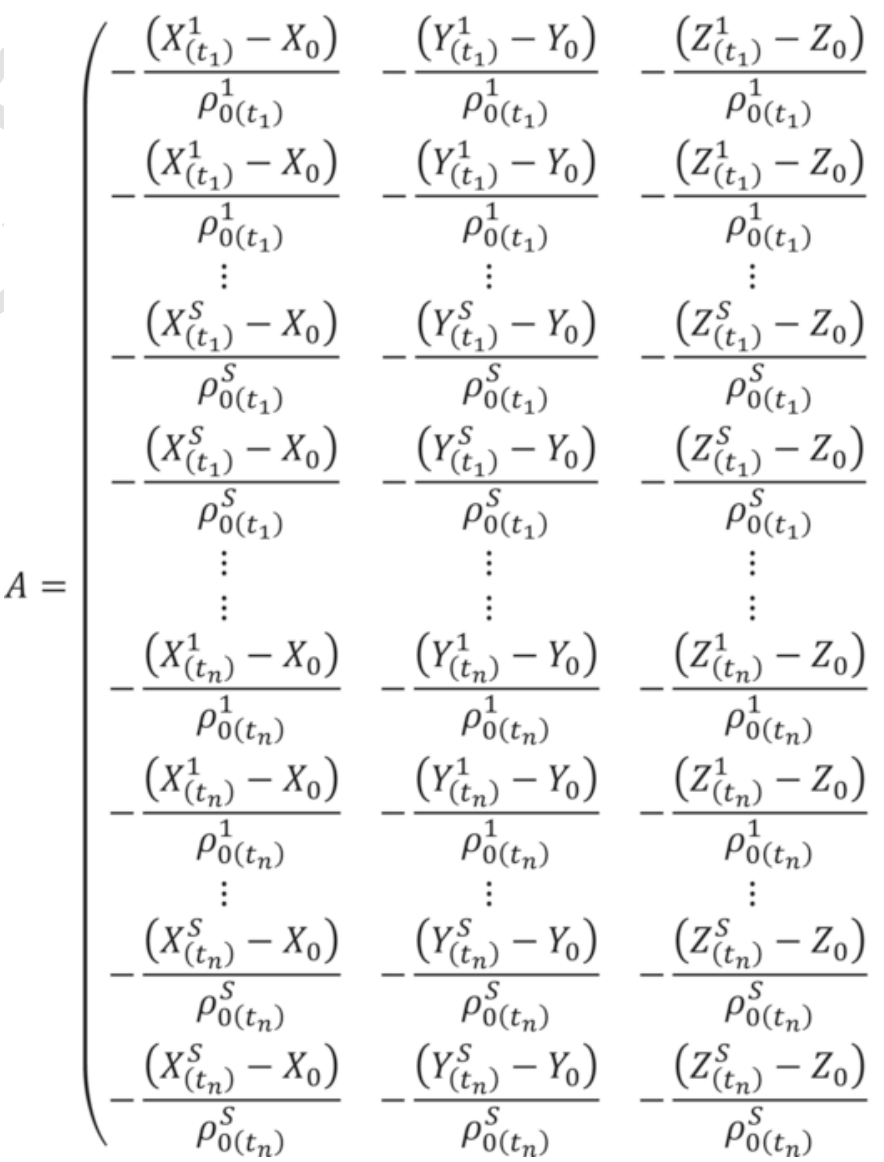

There are two equations for every satellite per epoch, the first corresponding to the pseudorange observation and the second to the carrier phase observation (containing the ambiguity term). $X_{0}, Y_{0}$ and $Z_{0}$ are the approximate coordinates of the station and $m$ is the mapping function of the tropospheric wet delay. The troposphere can 
be divided into two components, the hydrostatic component (dry) and wet component. The troposphere path delay can be expressed as a function of the dry and wet zenith path delay with an individual mapping function, relating the tropospheric delay to the elevation angle of the satellite. The hydrostatic component consists of mostly dry gases, is rather stable and can be modeled accurately with the Saastamoinen model. The wet component is a result of the water vapour, and it is difficult to model accurately, so in the linearization process of the observation equations, only the wet component is included as a parameter to be determined. The derivative with respect to the wet zenith path delay in the satellite direction is simply the mapping function of the wet part.

Matrix $b$ has the form

$b$

$$
=\left(\begin{array}{l}
P_{I F}^{1}\left(t_{1}\right)-\rho_{0}^{1}\left(t_{1}\right)+c d T^{1}\left(t_{1}\right)-c d t\left(t_{1}\right)-\operatorname{Trop}^{1}\left(t_{1}\right) \\
L_{I F}^{1}\left(t_{1}\right)-\rho_{0}^{1}\left(t_{1}\right)+c d T^{1}\left(t_{1}\right)-c d t\left(t_{1}\right)-\operatorname{Trop}^{1}\left(t_{1}\right) \\
\vdots \\
P_{I F}^{s}\left(t_{1}\right)-\rho_{0}^{s}\left(t_{1}\right)+c d T^{s}\left(t_{1}\right)-c d t\left(t_{1}\right)-\operatorname{Trop}^{s}\left(t_{1}\right) \\
L_{I F}^{s}\left(t_{1}\right)-\rho_{0}^{s}\left(t_{1}\right)+c d T^{s}\left(t_{1}\right)-c d t\left(t_{1}\right)-\operatorname{Trop}^{s}\left(t_{1}\right) \\
\vdots \\
\vdots \\
P_{I F}^{1}\left(t_{n}\right)-\rho_{0}^{1}\left(t_{n}\right)+c d T^{1}\left(t_{n}\right)-c d t\left(t_{n}\right)-\operatorname{Trop}^{1}\left(t_{n}\right) \\
L_{I F}^{1}\left(t_{n}\right)-\rho_{0}^{1}\left(t_{n}\right)+c d T^{1}\left(t_{n}\right)-c d t\left(t_{n}\right)-\operatorname{Trop}^{1}\left(t_{n}\right) \\
\vdots \\
P_{I F}^{s}\left(t_{n}\right)-\rho_{0}^{s}\left(t_{n}\right)+c d T^{s}\left(t_{n}\right)-c d t\left(t_{n}\right)-\operatorname{Trop}^{s}\left(t_{n}\right) \\
L_{I F}^{s}\left(t_{n}\right)-\rho_{0}^{s}\left(t_{n}\right)+c d T^{s}\left(t_{n}\right)-c d t\left(t_{n}\right)-\operatorname{Trop}^{s}\left(t_{n}\right)
\end{array}\right)
$$

where $P$ and $L$ are the observed ionosphere-free linear combinations of pseudrange and carrier phase respectively, ${ }_{0}$ is the geometric range receiver-satellite distance, $c d T$ is the satellite clock delay (obtained from the IGS clock final file), Trop is the tropospheric dry delay computed based on the Saastamoien zenith path delay and Niell mapping functions, $c d t$ is the a priori receiver clock correction, which is 0 for the first iteration of the first epoch, and, the computed value for the rest of the iterations; for the rest of the epochs, the value for the fist iteration is the final value obtained in the previous epoch, and, the computed value for the rest of the iterations.

Finally, the weight can be obtained as a function of elevation angle el corresponding to a satellite $1 \mathrm{~S}$ and epoch t:

$$
\begin{aligned}
W_{L} & =\frac{1}{e_{L}^{2} \cos \left(e l_{t}^{S}\right)^{2}} \\
W_{P} & =\frac{1}{e_{P}^{2} \cos \left(e l_{t}^{S}\right)^{2}}
\end{aligned}
$$

where $e_{P}$ is the a priori ionosphere-free standard deviation of pseudorange observations and $e_{L}$ is the ionosphere-free standard deviation of the phase observations. Typically, $e_{L}$ is $520 \mathrm{~mm}$ and the ratio $e_{P} / e_{L}=100$ for ionosphere-free undifferenced carrier phase and pseudorange observations is used.
Finally, the parameter vector is:

$X=\left(\begin{array}{l}\delta X \\ \delta Y \\ \delta Z \\ Z W D_{1} \\ \vdots \\ Z W D_{k} \\ c \Delta d t\left(t_{1}\right) \\ \vdots \\ c \Delta d t\left(t_{n}\right) \\ N_{I F}^{1} \\ \vdots \\ N_{I F}^{s}\end{array}\right)$

where $X, Y$ and $Z$ are the corrections to the a priori receiver position, $Z W D$ are the zenith components for the wet tropospheric delay ( $k$ different parameters, one every two hours), $c d t$ is the correction to the a priori receiver clock delay and $N_{I F}$ is the ionosphere-free ambiguity term for the satellites.

\section{Alternative method}

As previously mentioned, the main problem for the sequential batch solution of the system equations, is the amount of computer time it consumes, especially in computing $\left(A^{T} A\right)^{-1}$ as the number of equations increases. The basis for the time reduction begins with the known expression for matrix inversion (Strang and Borre, 1997):

$$
\left[\begin{array}{ll}
T & U \\
U^{T} & W
\end{array}\right]^{-1}=\left[\begin{array}{ll}
L & M \\
N & P
\end{array}\right]
$$

This block diagonal condition is true if the inverse matrix is the one needed to solve the least square PPP method (Eq. (5))

The method for obtaining this inverse is:

$$
L=T^{-1}+T^{-1} U P U^{T} T^{-1}
$$

$$
M=-T^{-1} U P
$$

$$
N=-P U^{T} T^{-1} P=\left(W-U^{T} T^{-1} U\right)^{-1}
$$

If, in the $U$ block, only parts of the normal equations coming from a new epoch are considered (i.e., clock delay; new troposphere delay, if it is necessary; or a new ambiguity term for any new satellites), the inverse can be obtained quickly from the inverse of the $T$ block, which is known from the previous epoch.

For the first epoch (ep1), matrix $T$ will be the usual system of normal equations $T_{e p 1}=\left(A_{e p 1}^{T} A_{e p 1}\right)$.

For the second epoch (ep2), the matrix $\left(A_{e p 2}^{T} A_{e p 2}\right)$ of normal equations, includes only the observations for this new epoch. Some of the unknown parameters are repeated from the previous epoch (i.e., receiver coordinates; troposphere delay, if this is the case; and ambiguity terms for repeated satellites), but the new unknown parameters define the $U$ matrix. The differential elements introduced for the new observations (epoch 2) in the system of normal equations defined in the first epoch, can be considered the sum of two matrices with the 
same columns (Shen and $\mathrm{Xu}, 2003$ ),

$$
\begin{aligned}
\left(A^{T} A\right)^{-1} & =\left(A_{e p 1 e p 2}^{T} A_{e p 1 e p 2}\right)^{-1} \\
& =\left(\begin{array}{c}
A_{e p 1}^{T} A_{e p 1} \\
A_{e p 2}^{T} A_{e p 2}
\end{array}\right)^{-1} \\
& =\left(A_{e p 1}^{T} A_{e p 1}+A_{e p 2}^{T} A_{e p 2}\right)^{-1}
\end{aligned}
$$

where the following expression can be used to solve the inverse:

$$
\begin{aligned}
(D+E C B)^{-1} & =D^{-1}-D^{-1} E K B D^{-1} K \\
& =\left(C^{-1}+B D^{-1} E\right)^{-1}
\end{aligned}
$$

In this expression, $C$ is the normal equation matrix generated for the second epoch, that is $C=\left(A_{e p 2}^{T} A_{e p 2}\right), D$ is the normal equations matrix from the previous epoch, that is $D=\left(A_{e p 1}^{T} A_{e p 1}\right)$, and $E$ and $B$ are defined such that they can be added to $D$. The only condition for this method is that $C$ and $D$ should be invertible matrices.

Obviously, $T$ block for the second epoch is $T=(D+E C B)$.

With this procedure, to solve the inverse of the normal equations matrix considering both epochs, it is necessary the inverted matrix of the normal equations computed in the previous epoch, which is known, and the inversion of the normal equation matrix generated only considering the observations of the second epoch, which is a small matrix.

For the third (and subsequent) epochs, the new matrix $D$ to add to the new matrix $C$ for the third epoch is the block matrix $\left[\begin{array}{cc}T & U \\ U^{T} & W\end{array}\right]$,obtained from the computation of the second epoch.

\section{Matrix design}

The main problem of this method is defining the design matrix $A$ and matrix $B$. In this section an example of the construction of the design

\begin{tabular}{|c|c|c|c|c|c|}
\hline & Station coordinates & & Tro & & \\
\hline$X$ & $\mathrm{Y}$ & $\mathrm{Z}$ & $\operatorname{Tr} 1$ & 0 & 1 \\
\hline $\mathrm{X}$ & $\mathrm{Y}$ & $\mathrm{Z}$ & $\operatorname{Tr} 1$ & 0 & 1 \\
\hline $\mathrm{X}$ & $\mathrm{Y}$ & $\mathrm{Z}$ & $\operatorname{Tr} 1$ & 0 & 1 \\
\hline $\mathrm{X}$ & $\mathrm{Y}$ & $\mathrm{Z}$ & $\operatorname{Tr} 1$ & 0 & 0 \\
\hline $\mathrm{X}$ & $\mathrm{Y}$ & $\mathrm{Z}$ & $\operatorname{Tr} 1$ & 0 & 0 \\
\hline $\mathrm{X}$ & $\mathrm{Y}$ & $\mathrm{Z}$ & $\operatorname{Tr} 1$ & 0 & 0 \\
\hline $\mathrm{X}$ & $\mathrm{Y}$ & $\mathrm{Z}$ & 0 & $\operatorname{Tr} 2$ & 0 \\
\hline $\mathrm{X}$ & $\mathrm{Y}$ & $\mathrm{Z}$ & 0 & $\operatorname{Tr} 2$ & 0 \\
\hline $\mathrm{X}$ & $\mathrm{Y}$ & $\mathrm{Z}$ & 0 & $\operatorname{Tr} 2$ & 0 \\
\hline $\mathrm{X}$ & $\mathrm{Y}$ & $\mathrm{Z}$ & 0 & $\operatorname{Tr} 2$ & 0 \\
\hline $\mathrm{X}$ & $\mathrm{Y}$ & $\mathrm{Z}$ & 0 & $\operatorname{Tr} 2$ & 0 \\
\hline $\mathrm{X}$ & $\mathrm{Y}$ & $\mathrm{Z}$ & 0 & $\operatorname{Tr} 2$ & 0 \\
\hline
\end{tabular}
matrix for the proposed method is described: imagine a matrix $A$ with for 4 epochs, 3 satellites and a change in the troposphere column, it will have the following simpified form (where only phase equations are considered for simplicity):

\begin{tabular}{|c|c|c|c|c|c|}
\hline $\mathrm{X}$ & Y & Z & $\operatorname{Tr} 1$ & 1 & 0 \\
\hline$X$ & $\mathrm{Y}$ & Z & $\operatorname{Tr} 1$ & 0 & 1 \\
\hline $\mathrm{X}$ & Y & Z & $\operatorname{Tr} 1$ & 0 & 0 \\
\hline $\mathrm{X}$ & Y & Z & $\operatorname{Tr} 1$ & 0 & 0 \\
\hline $\mathrm{X}$ & $\mathrm{Y}$ & Z & 0 & 0 & 1 \\
\hline $\mathrm{X}$ & $\mathrm{Y}$ & Z & 0 & 0 & 0 \\
\hline $\mathrm{X}$ & $\mathrm{Y}$ & Z & 0 & 0 & 0 \\
\hline $\mathrm{X}$ & Y & Z & 0 & 0 & 1 \\
\hline$X$ & $\mathrm{Y}$ & Z & 0 & 0 & 0 \\
\hline$X$ & $\mathrm{Y}$ & Z & 0 & 0 & 0 \\
\hline
\end{tabular}

However, the order in our method is different: new columns with the new parameters to be adjusted are added at the end of the matrix for every epoch, so, the same example will produce the following matrix:

\begin{tabular}{cccccr}
\hline & \multicolumn{2}{c}{ Station coordinates } & & Tropo & \multicolumn{2}{c}{ R.Clock } \\
\hline $\mathrm{X}$ & $\mathrm{Y}$ & $\mathrm{Z}$ & $\operatorname{Tr} 1$ & 1 & 1 \\
$\mathrm{X}$ & $\mathrm{Y}$ & $\mathrm{Z}$ & $\operatorname{Tr} 1$ & 1 & 0 \\
\hline
\end{tabular}

where the original block structure disappears and becomes sequential, and a new column is added if a new satellite, epoch or troposphere parameter appears.

The following example is used to explain the new matrix design process: imagine a first epoch with five satellites (satellites 15 ) and a second epoch with only two observed satellites: satellite 1 from the previous epoch and a new input, satellite 6 . The proposed design matrix $A$, considering only carrier-phase observations and one troposphere column, is:

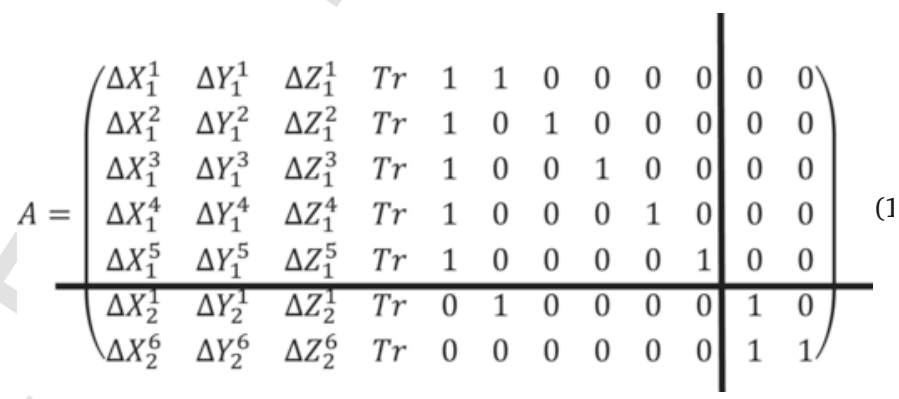

where the subscript is the epoch and the superscript is the satellite. Weight parameters are not included for simplicity, although the matrix design process is the same, per Eq. (5).

In block form:

$A=\left(\begin{array}{l|l}A_{11} & A_{12} \\ \hline A_{21} & A_{22}\end{array}\right)$

where the first block corresponds to the equations and parameters of the first epoch, and the remaining three blocks to the second epoch (the new receiver clock and ambiguity unknown for the satellite 6, which are reflected in the last two columns of the matrix).

The normal system of equations is:

$$
N=A^{T} A=
$$

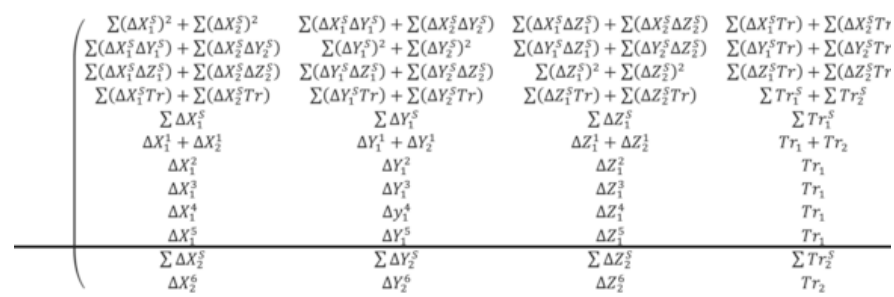


Thus, this is the matrix to invert per Eq. (10):

$N=\left[\begin{array}{ll}T & U \\ U^{T} & W\end{array}\right]^{-1}=\left[\begin{array}{ll}L & M \\ O & P\end{array}\right]$

Block $T$ is formed by the elements of the first epoch and the elements of the second epoch with the same unknowns, blocks $U$ and $W$ correspond to the two new unknowns columns (receiver clock epoch and ambiguity for satellite 6 , respectively). To solve this inversion, only the inverse of the $T$ block is needed, as in Eq. (11).

$T$ can be divided in two different matrices, one including only the elements of the first epoch and the second with elements of the second epoch with the same unknowns (the remaining elements are zero):

$T=\left(T_{1}+T_{2}\right)$
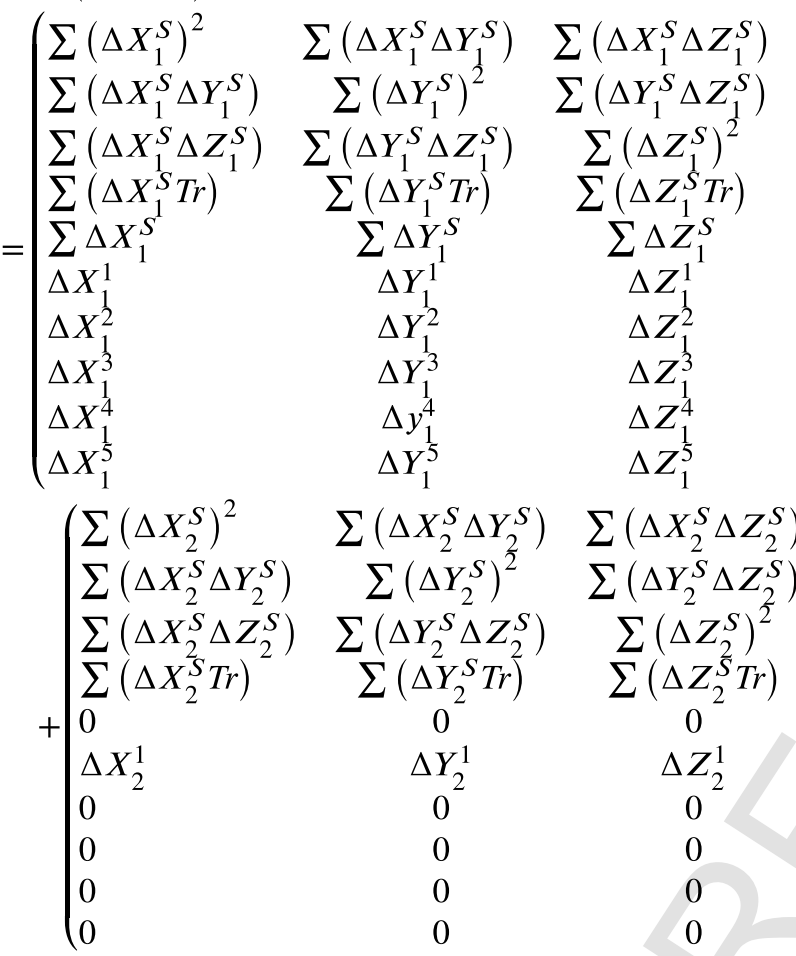

$\sum(\Delta$

$\sum(\Delta$

$=\mid \begin{aligned} & \sum\left(\Delta X^{S}\right. \\ & \sum \Delta X^{S} \\ & \sum X_{1}^{1} \\ & \Delta X_{1}^{2} \\ & \Delta X_{1}^{3} \\ & \Delta X_{1}^{4} \\ & \Delta X_{1}^{5}\end{aligned}$$$
\begin{aligned}
& \Delta y_{1}^{4} \\
& \Delta Y_{1}^{5}
\end{aligned}
$$

$\sum(\Delta$

$\Sigma\left(\Delta X_{2}^{S} \Delta Y_{2}^{S}\right)$

$\sum\left(\Delta X_{2}^{S} \Delta Z_{2}^{S}\right) \quad \sum$ $\sum\left(\Delta Y_{2}^{S}\right)^{2} \quad \sum\left(\Delta Y_{2}^{S} \Delta Z_{2}^{S}\right) \quad \sum$

Eq. (13) can be used to solve the inverse, in this case:

$$
\begin{aligned}
L & =\left(T_{1}+T_{2}\right)^{-1} \\
L & =\left(T_{1}+B C E\right)^{-1} \\
& =\left(T_{1}\right)^{-1}-\left(T_{1}\right)^{-1} B K E\left(T_{1}\right)^{-1} K \\
& =\left(C^{-1}+E\left(T_{1}\right)^{-1} B\right)^{-1}
\end{aligned}
$$

where $C$ is the normal equations generated in the second epoch and matrices $B$ and $E$ are defined so that the multiplication $B C E$ generates $T_{2}$. The only limitation is that $T_{1}$ and $C$ should be invertible matrices.
Following our example, matrix $C$ is:

C

$=\left(\begin{array}{lccr}\sum\left(\Delta X_{2}^{S}\right)^{2} & \sum\left(\Delta X_{2}^{S} \Delta Y_{2}^{S}\right) & \sum\left(\Delta X_{2}^{S} \Delta Z_{2}^{S}\right) & \sum(\Delta \\ \sum\left(\Delta X_{2}^{S} \Delta Y_{2}^{S}\right) & \sum\left(\Delta Y_{2}^{S}\right)^{2} & \sum\left(\Delta Y_{2}^{S} \Delta Z_{2}^{S}\right) & \sum(\Delta \\ \sum\left(\Delta X_{2}^{S} \Delta Z_{2}^{S}\right) & \sum\left(\Delta Y_{2}^{S} \Delta Z_{2}^{S}\right) & \sum\left(\Delta Z_{2}^{S}\right)^{2} & \sum(\Delta \\ \sum\left(\Delta X_{2}^{S} T r\right) & \sum\left(\Delta Y_{2}^{S} T r\right) & \sum\left(\Delta Z_{2}^{S} T r\right) & \sum \\ \sum \Delta X_{2}^{S} & \sum \Delta Y_{2}^{S} & \sum \Delta Z_{2}^{S} & \sum \\ \Delta X_{2}^{1} & \Delta Y_{2}^{1} & \Delta Z_{2}^{1} & \vdots \\ \Delta X_{2}^{6} & \Delta Y_{2}^{6} & \Delta Z_{2}^{6}\end{array}\right.$

Thus, the design of matrices $E$ and $B$ are simple in this example:

$$
E=\left(\begin{array}{cccccccccc|c}
a & a & a & b & c & d & e & e & e & e & \\
\hline 1 & 0 & 0 & 0 & 0 & 0 & 0 & 0 & 0 & 0 & a \\
0 & 1 & 0 & 0 & 0 & 0 & 0 & 0 & 0 & 0 & a \\
0 & 0 & 1 & 0 & 0 & 0 & 0 & 0 & 0 & 0 & a \\
0 & 0 & 0 & 1 & 0 & 0 & 0 & 0 & 0 & 0 & b \\
0 & 0 & 0 & 0 & 0 & 0 & 0 & 0 & 0 & 0 & c \\
0 & 0 & 0 & 0 & 0 & 1 & 0 & 0 & 0 & 0 & d \\
0 & 0 & 0 & 0 & 0 & 0 & 0 & 0 & 0 & 0 & e
\end{array}\right)
$$

and matrix $B$ is simply:

$B=E^{T}$

To clarify, the first three columns and rows of matrix $E$ ( $a$ columns and rows of Eq. (19)) correspond to the station coordinates, so the position of the ones will be always the same; the fourth column and row represents the troposphere, ( $b$ column and row of Eq. (19)). When a new column for the troposphere is considered (every two hours), this column is populated with zeros and a new column appears. The position of the new column is determined by the position of the new troposphere column in design matrix A, Eq. (14), (at the end of the matrix in our design). The next column and row is filled with zero and corresponds to the previous epoch (c column and row of Eq. (19)), corresponding with the receiver clock parameter of the first epoch; for the next epochs, because new parameters are added at the end of the system of equations, a new column of zeros is added at the end of matrix $E$. The last columns reference the satellites that appear in the observed epoch, if the satellite is repeated from previous observation (and no cycle slip has been determined) a column with a one in the corresponding row is introduced ( $d$ column and row of Eq. (19)) and for the rest of the satellites, the same number of columns as satellites are included, where the columns are full of zeros, but only one row is included ( $e$ columns and row of Eq. (19)). In this example, is easy to confirm that multiplying the defined matrices $B, C$ and $E$ generates matrix $T_{2}$.

$A_{12}$ and $A_{21}$ from Eq. (14b) can be easily obtained from matrix $C$ if we consider only the position of the new columns from the new epoch and satellite (the marked columns in matrix $C$, Eq. (20), repeated below for clarity). Finally, $A_{22}$ from Eq. (14b) is obtained using the intersecting elements of the columns and rows marked with a 
rectangle in matrix $C$ of Eq. (20).

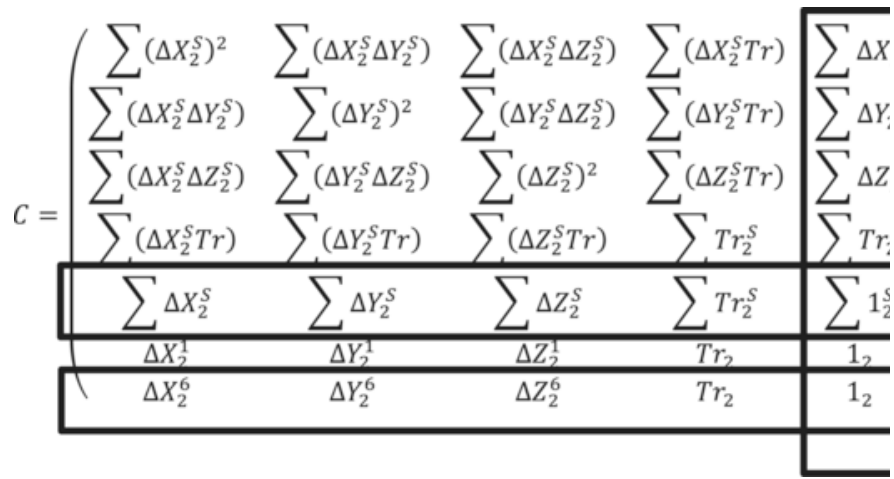

Therefore, the inverted matrix $T_{1}$ is needed to solve the inversion of Eq. (15), but this inversion has been done in the solution of the previous epoch in a sequential procedure. The inverse of $C$ and $K$ (Eq. (17)) must also be computed, but these are low-dimensional matrices (they contains only the satellites observed in the corresponding new epoch), so their inverse is not time consuming to compute.

\section{Tests}

The method described in the previous section has been implemented in the MATLAB source code of our PPP software.

In order to test the methodology, seven permanent IGS GNSS stations in North America (Fig. 2a) and four in Europe (Fig. 2b) were used, Table 1. Obervation occurred during $6 \mathrm{~h}$ (from 00:00 to 6:00) on October 3, 2013 , with a sample rate of $30 \mathrm{~s}$. The experiments involve comparing the time required to reach a PPP sequential solution with the traditional and proposed methodologies.

The same receiver coordinates, troposphere, receiver clock and ambiguities parameters were obtained for every epoch in each comparison between the traditional and proposed methodologies and all IGS stations. This indicate good numerical performance of the proposed methodology in comparison with the traditional one.

The mean reduction in computational time is $46 \%$, where the minimum is for SPT0 station with $36 \%$, and the maximum for ALGO with $54 \%$. The differences in the reduction time are related with the matrix dimension, a station with more observed satellites in comparison with another station, will produce a greater percentage of reduction in computational time.
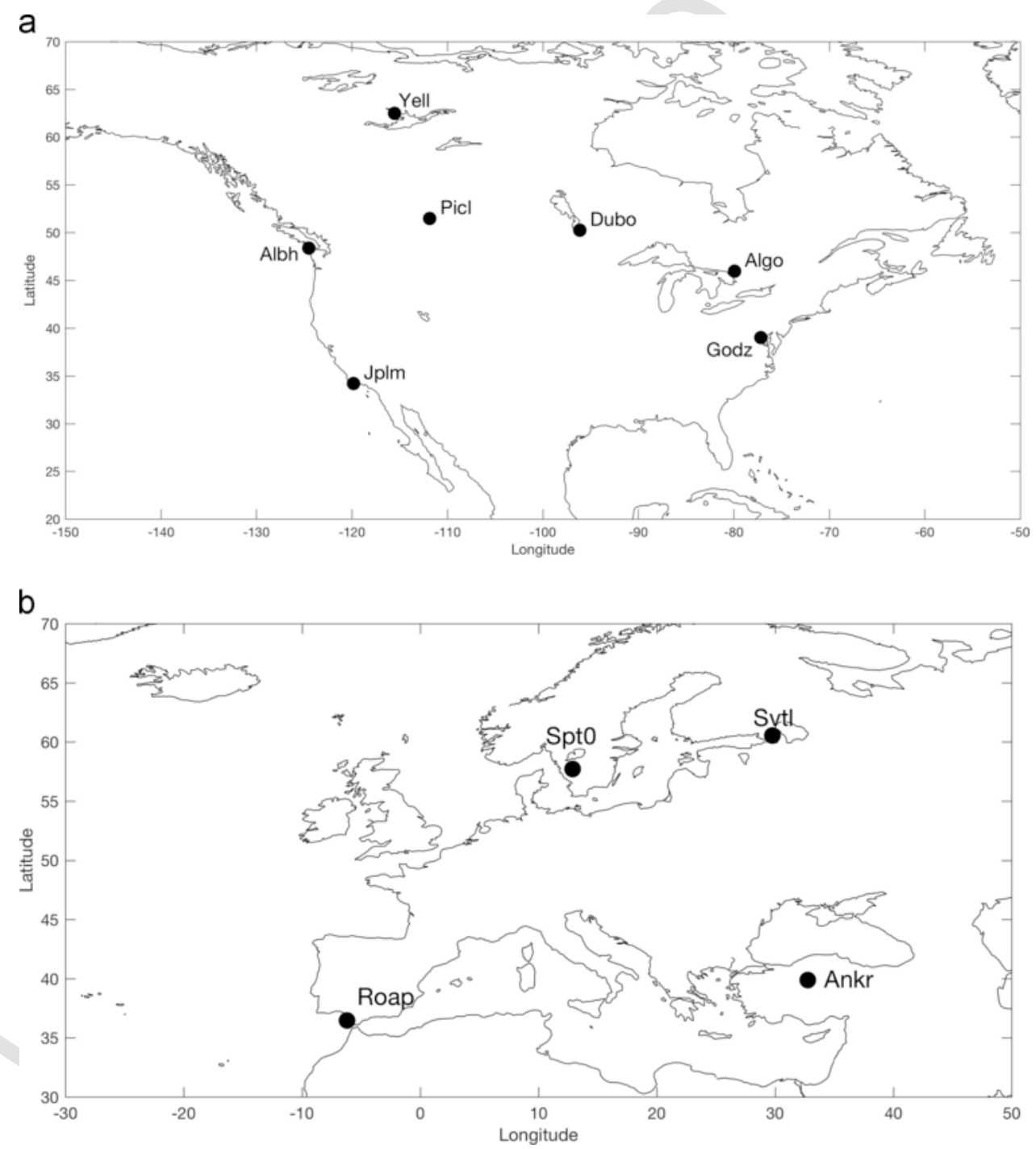

Fig. 2. (a) Location of the 7 North American IGS GNSS stations used in the study. Coastline file from the U.S. National Geophysical Data Center (2010). (b) Location of the 4 European IGS GNSS stations used in the study. Coastline file from the U.S. National Geophysical Data Center (2010). 
Table 1

Location, receivers, antennas and coordinates for the IGS permanent sites.

\begin{tabular}{|c|c|c|c|c|c|c|}
\hline Station & Location & Receiver & Antenna & Latitude (deg) & Longitude (deg) & $\mathrm{H}(\mathrm{m})$ \\
\hline ALBH & Victoria (Canada) & AOA B. ACT & AOAD/M_T SCIS & 48.389722 & -123.487222 & 32.0 \\
\hline ALGO & Algonqui-Park (Canada) & TPS NET-G3A & AOAD/M_T NONE & 45.958611 & -78.071388 & 202.0 \\
\hline ANKR & Ankara (Turkey) & TPS E_GGD & TPSCR3_GGD NONE & 39.887500 & 32.758333 & 974.8 \\
\hline DUBO & Lac Du Bonnet (Canada) & TPS NETG3 & AOAD/M_T NONE & 50.258611 & -95.866111 & 251.0 \\
\hline GODZ & Greenbelt (USA) & JPS EGGDT & AOAD/M_T JPLA & 39.021666 & -76.826666 & 14.5 \\
\hline JPLM & Pasadena (USA) & JPS EGGDT & AOAD/M_T NONE & 34.204722 & -118.173055 & 423.9 \\
\hline PICL & Pickle Lake (Canada) & AOA SNR-12 ACT & AOAD/M_T NONE & 51.479722 & -90.161944 & 315.1 \\
\hline ROAP & San Fernando (Spain) & SEPT POLARX3ETR & LEIAR25.R4 NONE & 36.464166 & -6.206111 & 73.7 \\
\hline SPTO & Boras (Sweden) & JAVAD TRE_G3TH & AOAD/M_T OSOD & 57.714722 & 12.891111 & 219.9 \\
\hline SVTL & Svetloe (Russian Federation) & TPS NETG3 & TPSCR.G3 TPSH & 60.532777 & 29.780833 & 77.1 \\
\hline YELL & Yellowknife (Canada) & JAVAD TRE_G3T & AOAD/M_T NONE & 62.480833 & -114.480555 & 181.0 \\
\hline
\end{tabular}

\section{Conclusions}

A method to reduce the time required to compute a sequential batch solution for static PPP was developed in this study. Our research is focused on the reduction of computation time in calculating the inverse matrix of the system of equations needed in the least-square solution. After theoretical development, the main difficulties occured in coding the method. The order of the columns, depending on new or previous parameters that arise, is the main factor to be considered in the matrix design process.

Finally, the developed method and software produce the same results as the traditional PPP method, but with a mean reduction in the computation time of $46 \%$. The method was tested using 11 IGS GNSS permanent stations.

This reduction in computational time can be used too if the interest are troposphere parameters for meteorogical purposes or receiver clock parameters for time transfer.

\section{Uncited references}

Shen and Xu (2013); Rai (2010).

\section{Acknowledgments}

This research was supported by the Spanish Science and Innovation Directorate project number AYA2010-18706 and the Generalitat Valenciana Ger nimo Forteza research program with project number FPA/2014/056.

We greatly appreciate the efforts of the IGS, Analysis and Data Centers and tracking station managers for generating high-quality data and products and for making them available to the GNSS community in a timely and reliable way.

The two anonymous reviewers are kindly acknowledged for their contribution to the improvement of the paper with their valuable comments and suggestions.

\section{References}

Bisnath, S., Gao, Y., 2008. Current state of precise point positioning and future prospects and limitations. In: Sideris, G. (Ed.), Proceedings of the Interna- tional Association of Geodesy Symposia 133: Observing our changing Earth, pp. 615623 . Chen W. Hu C. Gao S. Chen Y. Ding X. 2009. Error correction models and their effects on GPS Precise Point Positioning Surv. Rev. 41 (313), 238-252

Chen, K., 2004. Real-time precise point positioning and its potential application. In: Proceedings of the ION GNSS 17th International Technical Meeting of the Satellite Division. Long Beach, California.

Colombo, O.L., Sutter, A.W., Evans, A.G., 2004. Improving convergence sped of carrier phase based precise point positioning. In: Proceedings of the ION GNSS 17th International Technical Meeting of the Satellite Division of the Institute of Navigation. Institute of Navigation, Manassas, VA, pp. 14231430.

Dach, R., Lutz, S., Walser, P., Fridez P., 2015. Bernese GNSS Software Versi n 5.2. Draft, Astronomical Institute of Berne, Bern, Switzerland.

Dow, J.M., Neilan, R.E., Rizos, C., 2009. The international GNSS service in a changing landscape of global navigation satellite systems. J. Geod. 83 (3-4), 191-198.

Gao, Y., Shen, X., 2001. Improving convergence speed of carrier phase based precise point positioning. In: Proceedings of the ION GPS 14th International Technical Meeting of the Satellite Division of the Institute of Navigation. Institute of Navigation, Manassas, VA, pp. 15321539.

Geng, J., Meng, X., Teferle, N., Dodson, A., 2010. Performance of precise point positioning with ambiguity resolution for 1- to 4- hour observation period. Surv. Rev. 42 (316), $155-165$.

Heroux, P., Gao, Y., Kouba, J., Lahaye, F., Mireault, Y., Collins, P., Macleod, K., T treault, P., Chen, K., 2004. Products and applications for precise point positioning moving towards real-time. In: Proceedings of the ION GNSS 17th International Technical Meeting of the Satellite Division. Long Beach, California, pp. 18321843.

Hofmann-Wellenhof, B., Lichtenegger, H., Wasle, E., 2008. GNSS Global Navigation Satellite Systems. Springer Wien, New York, 2008.

IGS, 2013. IGS Quality of Service Fact Sheet. http://kb.igs.org/hc/en-us/articles/ 201208216-IGS-Quality-of-Service-Fact-Sheet , (Accessed 13 January 2017).

Kouba, J., H roux, P., 2001. Precise point positioning using IGS orbit and clock products. GPS Solut. 5 (2), 12-28.

Kouba, K., 2005. A possible detection of the 26 December 2004 great Sumatra-Andaman Islands earthquake with solution products of the international GNSS service. Stud. Geophys. Geod. 49 (4), 463-483.

Leick, A., 2004. GPS Satellite Surveying, third ed. Wiley, Hoboken.

Rai, J., 2010. [IGS-MAIL 6053]: Status of Orbits and Products. http://igscb.jpl.nasa.gov/ mail/igsmail/2010/msg00001.html .

Seepard, G., Bisnath, S., 2014. Challenges in assessing PPP performance. J. Appl. Geod. 8 (3), 205-222.

Shen, Y., Xu, G., 2013. Regularization and adjustment. In: Xu, G. (Ed.), Sciences of Geodesy II, Innovations and Future Developments. Springer-Verlag Berlin, Heidelberg, pp. 293-337.

Shi, J., Gao, Y., 2015. A comparison of three PPP integer ambiguity resolution methods. GPS Solut. 18 (4), 519-528.

Strang G. Borre K. 1997. Linear Algebra, Geodesy and GPS Wellesley-Cambridge Press

U.S. National Geophysical Data Center, 2010. Global Self-Consistent, Hierarchical, High-Resolution Shoreline Database (GSHHS). http://www.ngdc.noaa.gov/mgg/ shorelines/gshhs.html .

Zhang, X., Andersen, O.B., 2006. Surface ice flow velocity and tide retrieval of the Amery ice shelf using precise point positioning. J. Geod. 80 (4), 171-176.

Zumberge, J.F., Heflin, M.B., Jefferson, D.C., Watkins, M.M., Webb, F.H., 1997. Precise point positioning for the efficient and robust analysis of GPS data from large networks. J. Geophys. Res. 102 (B3), 5005-5018. 